\title{
Avaliação da qualidade da atenção ao aborto: protótipo de questionário para usuárias de serviços de saúde
}

\section{Quality assessment of treatment associated with abortion: a prototype questionnaire for health services users}

\section{Evaluación de la calidad en la atención al aborto: cuestionario prototipo para usuarias de los servicios de salud}

Estela M. L. Aquino 1

Greice M. S. Menezes 1

Thália V. Barreto-de-Araújo 2

Maria Teresa Alves 3

Maria da Conceição C. Almeida 4

Sandra Valongueiro Alves 2

Francisca Eleonora Schiavo 1

Lilian F. B. Marinho 5

Liberata C. Coimbra ${ }^{3}$

Michael E. Reichenheim 6

\author{
1 Instituto de Saúde Coletiva, \\ Universidade Federal da \\ Bahia, Salvador, Brasil. \\ 2 Programa de Pós-graduação \\ Integrado em Saúde Coletiva \\ Universidade Federal de \\ Pernambuco, Recife, Brasil. \\ 3 Programa de Pós-graduação \\ em Saúde Coletiva, \\ Universidade Federal do \\ Maranhão, São Luís, Brasil. \\ ${ }^{4}$ Centro de Pesquisas \\ Gonçalo Moniz, Fundação \\ Oswaldo Cruz, Salvador, \\ Brasil. \\ 5 Departamento de Ciência \\ da Vida, Universidade do \\ Estado da Bahia, Salvador \\ Brasil. \\ ${ }^{6}$ Instituto de Medicina \\ Social, Universidade do \\ Estado do Rio de Janeiro, \\ Rio de Janeiro, Brasil. \\ Correspondência \\ E. M. L. Aquino \\ Musa - Programa Integrado \\ em Gênero e Saúde, \\ Instituto de Saúde Coletiva \\ Universidade Federal da \\ Bahia. \\ Rua Basílio da Gama s/n, 2 o \\ andar, Salvador, \\ BA 40110-040, Brasil. \\ estela@ufba.br
}

\begin{abstract}
Little research in Brazil has focused on the quality of care following unsafe abortion. This article presents the first step in the development of an instrument to assess hospital care provided by the Brazilian Unified National Health System in three cities of Brazil. Along with related criteria, four key dimensions of care were defined: wellcome and guidance, technical quality of care, continuity of care, and supplies and physical environment. The authors performed a crosscultural adaptation of a set of items proposed by the World Health Organization. Following an assessment of the dimensions and criteria not captured by this set, the researchers decided to adapt questions from related studies and to add others developed by the research team itself. The questionnaire was pretested in 52 patients from three cities to assess the acceptance, understanding, and time of application and to make final adjustments. The instrument totaled 55 items organized according to different stages of care. Its expanded use depends on subsequent psychometric assessments, currently underway.

Induced Abortion; Health Services Evaluation; Questionnaires; Women's Health
\end{abstract}

\section{Resumo}

No Brasil, são escassas as pesquisas sobre a qualidade da atenção ao aborto inseguro. O presente artigo visa a apresentar a primeira etapa da construção de instrumento para estudo sobre a assistência hospitalar prestada pelo Sistema Único de Saúde em três cidades. Foram definidas quatro dimensões essenciais da atenção - acolhimento e orientação, qualidade técnica do cuidado, continuidade da atenção, insumos/ambiente físico - e respectivos critérios. Procedeu-se à adaptação transcultural de conjunto de itens propostos pela Organização Mundial da Saúde. Para dar conta de dimensões e critérios não contemplados pelo conjunto original de perguntas, optou-se por adaptar questões de outros estudos e adicionar outras elaboradas pela própria equipe. O questionário foi pré-testado em 52 usuárias, nas três cidades, para avaliar a aceitação e compreensão, o tempo de aplicação e ajustes finais. $O$ instrumento totalizou 55 itens, organizados segundo os momentos assistenciais, cujo uso mais amplo depende de avaliações psicométricas em desenvolvimento e que se seguirão em outros artigos.

Aborto Induzido; Avaliação de Serviços de Saúde; Questionários; Saúde da Mulher 


\section{Introdução}

No Brasil, são escassas as pesquisas sobre a qualidade da atenção ao aborto inseguro 1,2. Essa lacuna motivou a realização da pesquisa GravSus-NE sobre a assistência hospitalar ao aborto no Sistema Único de Saúde (SUS) em Salvador (Bahia), Recife (Pernambuco) e São Luís (Maranhão), cujos aspectos metodológicos e éticos encontram-se descritos em artigo publicado 3 .

A qualidade da atenção foi investigada tendo por referência o quadro ético-normativo da assistência integral à saúde das mulheres e ao abortamento em particular 4,5,6. Foram contempladas quatro dimensões essenciais da atenção - acolhimento e orientação, qualidade técnica do cuidado, continuidade da atenção e insumos / ambiente físico.

Acolhimento e orientação são entendidos como "o tratamento digno e respeitoso, a escuta, $o$ reconhecimento e a aceitação das diferenças, $o$ respeito ao direito de decidir de homens e mulheres" 6 (p. 17). A qualidade técnica do cuidado envolve a aplicação apropriada de conhecimento médico e tecnologia disponível, transformando recursos em resultados 7 . A continuidade do cuidado é orientada pelo princípio de integralidade da atenção e compreende o "conjunto articulado e contínuo das ações e serviços, preventivos e curativos" 8. A dimensão relativa a insumos/ambiente físico dos serviços remete às condições materiais necessárias à prestação adequada do cuidado.

Publicação recente 2 conclui pela avaliabilidade do modelo de atenção ao aborto proposto pelo Ministério da Saúde 6 . Contudo, não foram previamente identificados instrumentos para avaliar a qualidade desse tipo de atenção. Encontrou-se somente um documento da Organização Mundial da Saúde (OMS) ${ }^{9}$, que propunha itens para questionários sobre aborto inseguro, no qual se incluía módulo sobre qualidade da atenção em serviço de saúde, contemplando parcialmente as dimensões almejadas. As instruções de uso encorajavam sua ampla utilização mediante adaptações, o que motivou a construção de questionário adequado às especificidades nacionais e às normas brasileiras de atenção ao aborto 6 . O presente artigo visa a apresentar a primeira etapa da construção desse instrumento, prevendo testes e refinamento futuros.

\section{Métodos}

Iniciou-se pela atribuição de critérios e indicadores às quatro dimensões de qualidade consideradas para a avaliação ${ }^{3}$.
Posteriormente, procedeu-se à adaptação transcultural dos itens propostos pela OMS 9, os quais foram vertidos para o português, retrotraduzidos para o inglês e avaliados quanto à equivalência semântica 10. Duas traduções do inglês para o português foram efetuadas de modo independente por pesquisadoras versadas no tema, ambas brasileiras com proficiência em inglês. Após comparação das duas versões, elaborou-se versão de síntese, a qual foi retrotraduzida ao idioma original por pesquisadora inglesa com bom conhecimento de português. Outra pesquisadora inglesa com experiência no tema e proficiente em português fez avaliação independente e cega da equivalência semântica entre a versão retrotraduzida e o original.

A seguir, o conjunto de itens foi analisado com base nas quatro dimensões e respectivos critérios 3 . Para dar conta de dimensão não contemplada (insumos/ambiente físico) ou daquelas parcialmente tratadas (acolhimento e orientação; qualidade técnica do cuidado), optou-se por adaptar questões utilizadas em outros estudos 11,12 e adicionar perguntas elaboradas pela própria equipe.

O questionário foi pré-testado em 52 usuárias de serviços de atenção ao aborto, nas três cidades, a fim de avaliar a aceitação e compreensão das questões pelas entrevistadas, o tempo de aplicação, além de fazer ajustes finais.

\section{Resultados}

Os resultados da tradução/retrotradução e avaliação da equivalência semântica encontram-se na Tabela 1. Adaptações foram necessárias para acomodar diferenças culturais e institucionais. Por exemplo, excluiu-se "counselor" como alternativa de resposta para quem forneceu informações às usuárias, pela inexistência desse profissional no Brasil, e "parteira" como resposta à questão sobre responsável pelo esvaziamento uterino, pela ausência desse tipo de atuação em contextos urbanos brasileiros. Foram efetuadas substituições, como, por exemplo, "controle da fecundidade" por "planejamento familiar", devido ao uso consagrado deste último nos serviços de saúde; "receber contraceptivo" por "ter contraceptivo receitado”, em virtude de não ser habitual nos hospitais o fornecimento direto de métodos, mas sim sua prescrição. Alternativas sobre contraceptivos foram formatadas como na Pesquisa Nacional de Demografia e Saúde (PNDS-2006) 13, excluindo-se abstinência, coito interrompido e tabela. Foram excluídas duas questões abertas de interesse secundário que solicitavam sugestões para melhoria 
Itens propostos pela Organização Mundial da Saúde e versão adaptada para o português, segundo momento assistencial, dimensão e critério.

\begin{tabular}{|c|c|c|c|}
\hline \multirow{2}{*}{$\begin{array}{l}\text { Momento assistencial/ } \\
\text { Dimensão/Critério }\end{array}$} & \multirow[t]{2}{*}{ Itens na versão original 9} & \multicolumn{2}{|c|}{ Versão pós-tradução/retrotradução e equivalência semântica } \\
\hline & & Itens & Opções de resposta \\
\hline \multicolumn{4}{|l|}{ Antes do procedimento (A) } \\
\hline \multicolumn{4}{|l|}{ Acolhimento e orientação } \\
\hline Agilidade & $\begin{array}{l}\text { From the time that you arrived } \\
\text { at the hospital until the time that } \\
\text { someone first examined you, do } \\
\text { you think the waiting time was } \\
\text { appropriate or too long? }\end{array}$ & $\begin{array}{l}\text { Desde a hora em que chegou a } \\
\text { este hospital até a hora em que } \\
\text { alguém a examinou primeiro, você } \\
\text { acha que o tempo de espera foi } \\
\text { adequado ou longo demais? }\end{array}$ & $\begin{array}{c}\text { Adequado/Longo demais/Não tem } \\
\text { certeza }\end{array}$ \\
\hline Respeito * & $\begin{array}{l}\text { Did this person treat you } \\
\text { respectfully, with indifference, or } \\
\text { disrespectfully? }\end{array}$ & $\begin{array}{l}\text { Esta pessoa tratou você } \\
\text { respeitosamente, com indiferença } \\
\text { ou desrespeitosamente? }\end{array}$ & $\begin{array}{l}\text { Respeitosamente/Com indiferença/ } \\
\text { Desrespeitosamente/Outro. Qual? }\end{array}$ \\
\hline \multirow[t]{4}{*}{ Direito à informação } & $\begin{array}{l}\text { Did the person give you any } \\
\text { information about your health or } \\
\text { physical condition? }\end{array}$ & $\begin{array}{c}\text { Esta pessoa lhe deu alguma } \\
\text { informação sobre sua saúde ou sua } \\
\text { condição física? }\end{array}$ & Sim/Não \\
\hline & $\begin{array}{c}\text { Did you understand this } \\
\text { information? }\end{array}$ & Você entendeu esta informação? & Sim/Não/Não tem certeza \\
\hline & $\begin{array}{l}\text { Did the person give you any } \\
\text { information about what would } \\
\text { happen during the procedure? }\end{array}$ & $\begin{array}{l}\text { Esta pessoa lhe deu alguma } \\
\text { informação sobre o que } \\
\text { iria acontecer durante o } \\
\text { procedimento? }\end{array}$ & Sim/Não/Não lembra \\
\hline & $\begin{array}{l}\text { Did the person give you the } \\
\text { opportunity to ask questions? }\end{array}$ & $\begin{array}{l}\text { Esta pessoa lhe deu a } \\
\text { oportunidade de fazer perguntas? }\end{array}$ & Sim/Não/Não lembra \\
\hline \multicolumn{4}{|l|}{ Qualidade técnica do cuidado } \\
\hline Qualificação profissional & $\begin{array}{l}\text { Before the procedure, who } \\
\text { examined you? }\end{array}$ & $\begin{array}{c}\text { Antes do procedimento, quem } \\
\text { examinou você? }\end{array}$ & $\begin{array}{c}\text { Médico(a)/Enfermeira(o)/Parteira/ } \\
\text { Outro. Qual? }\end{array}$ \\
\hline \multicolumn{4}{|l|}{ Acolhimento e orientação } \\
\hline \multirow[t]{2}{*}{ Respeito * } & $\begin{array}{l}\text { Were you examined by a man or a } \\
\text { woman? }\end{array}$ & $\begin{array}{l}\text { Você foi examinada por um } \\
\text { homem ou uma mulher? }\end{array}$ & Homem/Mulher \\
\hline & What would be your preference? & $\begin{array}{l}\text { Se pudesse escolher, qual teria } \\
\text { sido sua preferência? }\end{array}$ & Homem/Mulher/Indiferente \\
\hline \multicolumn{4}{|l|}{ Durante o procedimento (D) } \\
\hline \multicolumn{4}{|l|}{ Acolhimento e orientação } \\
\hline Agilidade & $\begin{array}{l}\text { From the time that you were } \\
\text { first examined until the time the } \\
\text { procedure began, was your wait } \\
\text { appropriate or too long? }\end{array}$ & $\begin{array}{c}\text { Desde a hora em que alguém a } \\
\text { examinou primeiro até a hora em } \\
\text { que começou o procedimento, o } \\
\text { seu tempo de espera foi adequado } \\
\text { ou muito longo? }\end{array}$ & $\begin{array}{c}\text { Adequado/Longo demais/Não tem } \\
\text { certeza }\end{array}$ \\
\hline \multirow[t]{2}{*}{ Respeito * } & $\begin{array}{l}\text { Did this person treat you } \\
\text { respectfully, with indifference, or } \\
\text { disrespectfully? }\end{array}$ & $\begin{array}{l}\text { Esta pessoa tratou você } \\
\text { respeitosamente, com indiferença } \\
\text { ou desrespeitosamente? }\end{array}$ & $\begin{array}{l}\text { Respeitosamente/Com indiferença/ } \\
\text { Desrespeitosamente/Outro. Qual? }\end{array}$ \\
\hline & $\begin{array}{l}\text { Did the person treating you } \\
\text { console you during the procedure? }\end{array}$ & $\begin{array}{l}\text { A pessoa que estava cuidando de } \\
\text { você, confortou você durante o } \\
\text { procedimento? }\end{array}$ & Sim/Não/Não lembra \\
\hline \multirow[t]{3}{*}{ Direito à informação } & $\begin{array}{l}\text { Did the person explain what was } \\
\text { happening during the procedure? }\end{array}$ & $\begin{array}{c}\text { Esta pessoa explicou o que } \\
\text { estava acontecendo durante o } \\
\text { procedimento? }\end{array}$ & Sim/Não/Não lembra \\
\hline & $\begin{array}{c}\text { Did you understand the information } \\
\text { that you were given? }\end{array}$ & $\begin{array}{l}\text { Você entendeu a informação que } \\
\text { Ihe foi dada? }\end{array}$ & Sim/Não/Não lembra \\
\hline & $\begin{array}{l}\text { Did you want to know what was } \\
\text { happening during the procedure? }\end{array}$ & $\begin{array}{c}\text { Você quis saber o que estava } \\
\text { acontecendo durante o } \\
\text { procedimento? }\end{array}$ & Sim/Não/Não lembra \\
\hline
\end{tabular}

(continua) 
Tabela 1 (continuação)

\begin{tabular}{|c|c|c|c|}
\hline \multirow{2}{*}{$\begin{array}{l}\text { Momento assistencial/ } \\
\text { Dimensão/Critério }\end{array}$} & \multirow[t]{2}{*}{ Itens na versão original 9} & \multicolumn{2}{|c|}{ Versão pós-tradução/retrotradução e equivalência semântica } \\
\hline & & Itens & Opções de resposta \\
\hline \multicolumn{4}{|l|}{ Qualidade técnica do cuidado } \\
\hline Integridade física & $\begin{array}{c}\text { Were you awake or asleep during } \\
\text { the procedure? }\end{array}$ & $\begin{array}{c}\text { Você estava acordada ou dormindo } \\
\text { durante o procedimento? }\end{array}$ & Acordada/Dormindo \\
\hline Qualificação profissional & Who performed the procedure? & Quem realizou o procedimento? & Médico(a)/Outro. Qual?/Não sabe \\
\hline \multirow[t]{2}{*}{ Integridade física } & $\begin{array}{c}\text { Did you feel any pain during the } \\
\text { procedure? }\end{array}$ & $\begin{array}{c}\text { Você sentiu alguma dor durante o } \\
\text { procedimento? }\end{array}$ & $\operatorname{Sim} /$ Não \\
\hline & $\begin{array}{c}\text { Were you given any medication to } \\
\text { ease the pain? }\end{array}$ & $\begin{array}{l}\text { Deram a você alguma medicação } \\
\text { para aliviar a dor? }\end{array}$ & Sim/Não/Não lembra \\
\hline \multicolumn{4}{|l|}{ Pós-procedimento (P) } \\
\hline \multicolumn{4}{|l|}{ Acolhimento e orientação } \\
\hline \multirow[t]{2}{*}{ Respeito * } & $\begin{array}{c}\text { Were you treated respectfully, with } \\
\text { indifference, or disrespectfully by } \\
\text { this person? }\end{array}$ & $\begin{array}{c}\text { Você foi tratada respeitosamente, } \\
\text { com indiferença ou } \\
\text { desrespeitosamente por } \\
\text { esta pessoa? }\end{array}$ & $\begin{array}{l}\text { Respeitosamente/Com indiferença/ } \\
\text { Desrespeitosamente/Outro. Qual? }\end{array}$ \\
\hline & $\begin{array}{l}\text { Did the person who gave } \\
\text { you information treat you } \\
\text { respectfully, with indifference, or } \\
\text { disrespectfully? }\end{array}$ & $\begin{array}{l}\text { A pessoa que lhe deu informação } \\
\text { tratou você respeitosamente, } \\
\text { com indiferença ou } \\
\text { desrespeitosamente? }\end{array}$ & $\begin{array}{l}\text { Respeitosamente/Com indiferença/ } \\
\text { Desrespeitosamente/Outro. Qual? }\end{array}$ \\
\hline Direito à informação & $\begin{array}{c}\text { Were you encouraged to ask } \\
\text { questions? }\end{array}$ & $\begin{array}{l}\text { Você foi encorajada a } \\
\text { fazer perguntas? }\end{array}$ & Sim/Não \\
\hline \multicolumn{4}{|l|}{ Qualidade técnica } \\
\hline \multirow[t]{2}{*}{ Qualificação profissional } & Who gave you this information? & $\begin{array}{l}\text { Quem Ihe forneceu } \\
\text { esta informação? }\end{array}$ & $\begin{array}{c}\text { Médico(a)/Enfermeira(o)/Parteira/ } \\
\text { Assistente Social/Outra pessoa. } \\
\text { Qual? }\end{array}$ \\
\hline & $\begin{array}{c}\text { Who examined you after the } \\
\text { procedure? }\end{array}$ & $\begin{array}{l}\text { Quem examinou você após o } \\
\text { procedimento? }\end{array}$ & $\begin{array}{c}\text { Médico(a)/Enfermeira(o)/Parteira/ } \\
\text { Ninguém/Outro. Qual? }\end{array}$ \\
\hline \multicolumn{4}{|l|}{ Continuidade do cuidado ** } \\
\hline Cuidados pós-alta & $\begin{array}{c}\text { Did this person explain how to } \\
\text { care for yourself after leaving the } \\
\text { hospital? }\end{array}$ & $\begin{array}{c}\text { Alguém explicou os cuidados que } \\
\text { você deveria ter após } \\
\text { sair do hospital? }\end{array}$ & Sim/Não/Não lembra \\
\hline Orientações sobre gravidez & $\begin{array}{c}\text { Did the person explain that you } \\
\text { can immediately become pregnant } \\
\text { once again? }\end{array}$ & $\begin{array}{c}\text { Alguém explicou que você } \\
\text { poderia imediatamente ficar } \\
\text { grávida de novo? }\end{array}$ & Sim/Não/Não lembra \\
\hline Consulta de revisão & $\begin{array}{l}\text { Do you have an appointment for a } \\
\text { follow-up visit? }\end{array}$ & $\begin{array}{c}\text { Foi marcado algum retorno ou } \\
\text { alguma consulta de revisão } \\
\text { após sua alta? }\end{array}$ & Sim/Não \\
\hline Planejamento reprodutivo & $\begin{array}{c}\text { Were you given any information } \\
\text { about } \\
\text { fertility control? }\end{array}$ & $\begin{array}{c}\text { Deram a você alguma informação } \\
\text { sobre planejamento familiar? }\end{array}$ & Sim/Não/Não lembra \\
\hline \multirow[t]{3}{*}{ Acesso à contracepção } & $\begin{array}{l}\text { Were you given a modern } \\
\text { contraceptive method? }\end{array}$ & $\begin{array}{c}\text { Receitaram a você algum método } \\
\text { contraceptivo? }\end{array}$ & Sim/Não \\
\hline & What method did you choose? & Qual método foi receitado? & $\begin{array}{c}\text { Pílula/Injeções/Implantes } \\
\text { (Norplant)/DIU/Diafragma/ } \\
\text { Camisinha masculina/Camisinha } \\
\text { feminina/Outro. Qual?/Não sabe } \\
\text { ou não lembra }\end{array}$ \\
\hline & $\begin{array}{l}\text { Were you told where you could get } \\
\text { contraceptives in your community? }\end{array}$ & $\begin{array}{l}\text { Falaram para você onde poderia } \\
\text { conseguir contraceptivos? }\end{array}$ & Sim/Não \\
\hline
\end{tabular}

Fonte: Organização Mundial da Saúde 9.

* Respeito à condição de pessoa e/ou direito à escolha;

** Os critérios desta dimensão podem ser também classificados como direito à informação, respeito à condição de pessoa; direito à escolha; componentes da atenção. 
do atendimento, cuja análise seria dificultada pelo grande número de entrevistadas.

A Tabela 2 mostra os itens elaborados pela equipe de pesquisa ou os adaptados de outras investigações: privacidade; presença de acompanhante; tipo de procedimento de esvaziamento uterino; existência, intensidade e manejo da dor; além de outros relativos à qualidade técnica do cuidado. Foi incluído item inquirindo se as usuárias haviam sido consultadas sobre o método contraceptivo de escolha, por ser frequente a prescrição sem consulta à preferência das mulheres. Também foi incluído item para sintetizar a satisfação com o atendimento, informação importante para avaliar a qualidade da atenção na perspectiva da humanização 14. Para mensurar a dor após o esvaziamento uterino, adotou-se escala visual analógica, já validada no Brasil 15.

O instrumento final (Tabela 3) totalizou 55 itens, organizados segundo os diferentes momentos assistenciais: antes, durante e após o esvaziamento uterino; atenção pós-alta; impressões gerais sobre o atendimento. Os dois últimos devem ser aplicados após a alta médica.

O pré-teste evidenciou boa aceitação pelas entrevistadas. Não houve maiores dificuldades de compreensão, exceto quanto ao termo "procedimento", o que motivou a incorporação de instrução à entrevistadora para citar diretamente o tipo de técnica (se aspiração manual intrauterina ou curetagem).

\section{Discussão}

Não é pequeno o desafio de proceder à adaptação transcultural de instrumentos concebidos para contextos diversos. Os itens propostos pela OMS ${ }^{9}$ não constituíam instrumento validado, mas serviram como ponto de partida para a elaboração de questionário culturalmente adequado para usuárias avaliarem a qualidade da atenção.
Na maioria das questões são solicitadas informações factuais e objetivas, que incluem a realização de tecnologias de baixa densidade e conhecimento universalmente disseminado, como a aferição de temperatura corporal e pressão arterial ou o controle do sangramento. Entretanto, itens que avaliam a percepção de usuárias sobre as relações interpessoais e a humanização da atenção 14 envolvem grande subjetividade e devem ser objeto de cuidadosa avaliação psicométrica. A satisfação com o atendimento, por exemplo, reflete experiências passadas de situações similares, vivenciadas pela própria mulher ou por familiares e conhecidas, moldando a expectativa das usuárias 14.

A adoção quase exclusiva da curetagem para esvaziamento uterino ${ }^{3}$ compromete a aplicação dos itens que avaliam este momento assistencial, já que as mulheres encontram-se sedadas durante o procedimento. Tal quadro, por si só, indica pior qualidade da atenção ${ }^{6}$.

A qualidade da informação depende da memória, que, por sua vez, é influenciada pelas condições da rememoração ${ }^{14}$. Idealmente, o questionário deveria ser aplicado fora do ambiente hospitalar, especialmente pela possibilidade do viés de gratidão 14 . Contudo, em face de dificuldades para localizar as mulheres a serem entrevistadas após a alta hospitalar, sua aplicação no hospital torna-se imperiosa para evitar perdas. Recomenda-se que as entrevistas sejam realizadas após a curetagem e, obrigatoriamente, após a alta médica, quando as mulheres se encontram em bom estado físico, sem dor e aguardam os procedimentos administrativos para sua liberação.

Por fim, cabe enfatizar que o instrumento deve ser complementado por quesitos marcadores da posição social das mulheres, tais como idade, raça/cor, escolaridade e renda, que permitam apreender desigualdades sociais na prestação do cuidado. Sua utilização mais ampla depende ainda de extensas análises, desde avaliações de estrutura dimensional até estudos de validação de construto via testes de hipótese 16 . Nesse sentido, avaliações psicométricas estão em desenvolvimento e se seguirão em outros artigos. 
Tabela 2

Itens desenvolvidos ou adaptados de outros instrumentos 11,12, segundo momento assistencial, dimensão, critério e fonte.

\begin{tabular}{|c|c|c|c|}
\hline $\begin{array}{l}\text { Momento assistencial/ } \\
\text { Dimensão/Critério }\end{array}$ & Item & Opções de resposta & Fonte \\
\hline \multicolumn{4}{|l|}{ Antes do procedimento (A) } \\
\hline \multicolumn{4}{|l|}{ Acolhimento e orientação } \\
\hline \multirow[t]{3}{*}{ Privacidade } & $\begin{array}{c}\text { Durante o exame, havia outras pessoas } \\
\text { presentes? * }\end{array}$ & Sim/Não/Não lembra & Equipe \\
\hline & Quem eram essas pessoas? & $\begin{array}{l}\text { Profissionais de saúde (médicos, pessoal } \\
\text { de enfermagem, residentes etc.)/Pacientes/ } \\
\text { Acompanhantes das pacientes/Não sabe }\end{array}$ & Equipe \\
\hline & $\begin{array}{l}\text { A presença de pessoas além dos profissionais } \\
\text { de saúde que estavam examinando você lhe } \\
\text { causou constrangimento (ou vergonha)? }\end{array}$ & $\begin{array}{l}\text { Muito constrangimento/Um pouco de } \\
\text { constrangimento/Não/Não lembra }\end{array}$ & Equipe \\
\hline \multicolumn{4}{|l|}{ Qualidade técnica do cuidado } \\
\hline $\begin{array}{l}\text { Técnica de esvaziamento } \\
\text { uterino }\end{array}$ & $\begin{array}{c}\text { Durante esta internação, você realizou uma } \\
\text { curetagem ou AMIU para esvaziamento do } \\
\text { útero? }\end{array}$ & $\begin{array}{l}\text { Curetagem/AMIU/Sim, mas não sabe qual } \\
\text { procedimento/Não }\end{array}$ & Equipe \\
\hline \multirow[t]{2}{*}{ Integridade física } & $\begin{array}{l}\text { Você sentiu alguma dor antes de realizar o } \\
\quad<\text { citar o procedimento realizado>? }\end{array}$ & Sim/Não & Equipe \\
\hline & $\begin{array}{c}\text { Deram a você alguma medicação para aliviar } \\
\text { essa dor? }\end{array}$ & Sim/Não/Não lembra & Equipe \\
\hline \multicolumn{4}{|l|}{ Durante o procedimento (D) } \\
\hline \multicolumn{4}{|l|}{ Acolhimento e orientação } \\
\hline \multirow[t]{2}{*}{ Apoio social } & $\begin{array}{l}\text { Seu companheiro, ou alguma pessoa da } \\
\text { família ou amiga, ficou com você durante o } \\
\text { tempo em que esteve internada? }\end{array}$ & $\begin{array}{c}\text { Sim, durante toda internação/ } \\
\text { Sim, apenas parte do tempo/Não }\end{array}$ & Adaptado 12 \\
\hline & Por que não teve acompanhante? & $\begin{array}{l}\text { Não é permitido pelo hospital/ } \\
\text { Não é permitido acompanhante homem/ } \\
\text { Desconhecia que era permitido/ } \\
\text { Não tinha acompanhante disponível/ } \\
\text { Não teve necessidade/ } \\
\text { Não quis acompanhante/Outro. Qual? }\end{array}$ & Adaptado 12 \\
\hline \multirow[t]{2}{*}{ (não discriminação) } & $\begin{array}{c}\text { Você acha que algum profissional de saúde } \\
\text { deste hospital - médico/a, enfermeira/o ou } \\
\text { outro - Ihe deu atendimento pior do que às } \\
\text { outras pacientes? }\end{array}$ & Sim/Não/Não lembra & Adaptado 12 \\
\hline & Por que você acha que isso aconteceu? & $\begin{array}{l}\text { Pela sua idade (por ser muito jovem ou mais } \\
\text { velha)/Pela cor da sua pele ou sua raça/Pela } \\
\text { sua condição econômica/ } \\
\text { Pela sua instrução/Pela sua religião ou culto/ } \\
\text { Por ser mulher/Por acharem que o aborto foi } \\
\text { provocado/Outro motivo. Qual? }\end{array}$ & Adaptado 12 \\
\hline \multicolumn{4}{|l|}{ Qualidade técnica do cuidado } \\
\hline \multirow[t]{2}{*}{ Integridade física } & $\begin{array}{l}\text { Você sentiu alguma dor durante o } \\
\text { procedimento? }\end{array}$ & Sim/Não & Equipe \\
\hline & $\begin{array}{c}\text { Deram a você alguma medicação para aliviar } \\
\text { essa dor? }\end{array}$ & Sim/Não/Não lembra & Equipe \\
\hline
\end{tabular}

(continua) 


\begin{tabular}{l} 
Momento assistencial/ \\
Dimensão/Critério \\
\hline Pós-procedimento (P) \\
Acolhimento e orientação \\
Privacidade
\end{tabular}

Privacidade

Qualidade técnica do cuidado Integridade física

\section{Acolhimento e orientação Respeito *}

Impressão geral do atendimento durante toda internação (IGA)

Qualidade técnica do cuidado Controle de pressão arterial

Controle de temperatura

Controle de sangramento

Insumos/Ambiente físico

Limpeza de roupa de cama

Limpeza do ambiente

Roupa adequada

Absorventes suficientes
Durante o exame, havia outras pessoas presentes?*

Quem eram essas pessoas?

A presença de pessoas além dos profissionais de saúde que a estavam examinando the causou constrangimento (ou vergonha)?

Agora, queremos saber se depois que você realizou <citar o procedimento realizado>, sentiu dor e qual a intensidade da dor sentida.

Deram a você alguma medicação para aliviar essa dor?

Você foi consultada sobre o método de sua preferência?

Durante a sua internação, algum profissional de saúde mediu a sua pressão?

Durante a sua internação, algum profissional de saúde tirou (ou mediu) a sua temperatura?

Durante a sua internação, algum profissional de saúde avaliou a quantidade de seu sangramento?

A troca de roupa de cama foi:

Você considera a limpeza das enfermarias e outros ambientes do hospital, incluindo os banheiros, como:

A roupa que foi fornecida para você vestir foi:

O fornecimento de absorventes para você trocar foi:
Sim/Não/Não lembra

Equipe

Profissionais de saúde (médicos, pessoal de enfermagem, residentes etc)/Pacientes/ Acompanhantes das pacientes/Não sabe

Muito constrangimento/Um pouco de constrangimento/Não/Não lembra

Para isso, dê uma nota de zero a dez para a intensidade da dor. (Apresente a cartela para classificação da dor e registre a nota dada.)

Sim/Não/Não lembra

Equipe

Sim/Não

Equipe

Não/Sim, antes de realizar < citar o procedimento realizado $>/$ Sim, depois de realizar <citar o procedimento realizado $>$ / Sim, antes e depois de realizar <citar o procedimento realizado $>$ /Não lembra

Não/Sim, antes de realizar < citar o procedimento realizado $>/$ Sim, depois de realizar <citar o procedimento realizado>/ Sim, antes e depois de realizar < citar o procedimento realizado>/Não lembra

Não/Sim, antes de realizar <citar o procedimento realizado $>/$ Sim, depois de realizar <citar o procedimento realizado>/ Sim, antes e depois de realizar <citar o procedimento realizado $>$ /Não lembra

Suficiente/Insuficiente/Não houve troca/Não tinha roupa de cama

Excelente/Boa/Regular/Ruim/Péssima/Não tem opinião formada

Muito grande/Grande/Do tamanho adequado/Pequena /Muito pequena/Não foi fornecida roupa

Suficiente (sempre que precisou)/Regular/ Insuficiente/Não tem opinião formada
Adaptado 11

Adaptado 11

Equipe

Adaptado 11

Adaptado 12

Equipe

Equipe

AMIU: aspiração manual intrauterina.

Fontes: Silva et al. 11; Organização Mundial da Saúde 12.

* Respeito ao direito à escolha. 
Tabela 3

Protótipo de instrumento construído para avaliação da qualidade da atenção ao aborto na perspectiva das usuárias, GravSus-NE.

\begin{tabular}{|c|c|c|c|}
\hline $\begin{array}{l}\text { Momento assistencial/ } \\
\text { Dimensão/Critério }\end{array}$ & $\begin{array}{l}\text { Número } \\
\text { do item }\end{array}$ & Indicador & Fonte \\
\hline \multicolumn{4}{|l|}{ Antes do procedimento $(A)$} \\
\hline \multicolumn{4}{|l|}{ Acolhimento e orientação } \\
\hline \multirow[t]{2}{*}{ Agilidade } & A1 & Tempo de espera adequado & OMS \\
\hline & & (desde a chegada ao hospital até o primeiro exame) & \\
\hline \multicolumn{4}{|l|}{ Qualidade técnica do cuidado } \\
\hline Técnica de esvaziamento uterino & A2 & Tipo de procedimento para esvaziamento do útero & Equipe \\
\hline \multicolumn{4}{|l|}{ Qualidade técnica do cuidado } \\
\hline Qualificação profissional & A3 & Tipo de profissional que realizou o exame antes do procedimento & OMS \\
\hline \multicolumn{4}{|l|}{ Acolhimento e orientação } \\
\hline Respeito * & A4 & Sexo do profissional que realizou o exame antes do procedimento & OMS \\
\hline \multicolumn{4}{|l|}{ Acolhimento e orientação } \\
\hline Respeito * & A5 & $\begin{array}{l}\text { Preferência pelo sexo do profissional que realizou o } \\
\text { exame antes do procedimento }\end{array}$ & OMS \\
\hline \multicolumn{4}{|l|}{ Acolhimento e orientação } \\
\hline Respeito* & A6 & Tratamento respeitoso (durante o exame antes do procedimento) & OMS \\
\hline \multicolumn{4}{|l|}{ Acolhimento e orientação } \\
\hline Direito à informação & A7 & Receberam informação sobre saúde/condição física & OMS \\
\hline \multicolumn{4}{|l|}{ Acolhimento e orientação } \\
\hline Direito à informação & A8 & Entenderam a informação sobre saúde/condição física & OMS \\
\hline \multicolumn{4}{|l|}{ Acolhimento e orientação } \\
\hline Direito à informação & A9 & Receberam informação sobre o procedimento de esvaziamento uterino & OMS \\
\hline \multicolumn{4}{|l|}{ Acolhimento e orientação } \\
\hline Direito à informação & A10 & $\begin{array}{l}\text { Tiveram oportunidade de fazer perguntas ao } \\
\text { receberem informações sobre o procedimento }\end{array}$ & OMS \\
\hline \multicolumn{4}{|l|}{ Acolhimento e orientação } \\
\hline Privacidade & A11 & Presença de pessoas durante exame (antes do procedimento) & Equipe \\
\hline \multicolumn{4}{|l|}{ Acolhimento e orientação } \\
\hline Privacidade & A12 & Tipo de pessoas presentes durante exame (antes do procedimento) & Equipe \\
\hline \multicolumn{4}{|l|}{ Acolhimento e orientação } \\
\hline Privacidade & A13 & $\begin{array}{l}\text { Constrangimento pela presença de pessoas durante exame } \\
\qquad \text { (antes do procedimento) }\end{array}$ & Equipe \\
\hline \multicolumn{4}{|l|}{ Qualidade técnica do cuidado } \\
\hline Integridade física & A14 & Dor antes do procedimento & Equipe \\
\hline \multicolumn{4}{|l|}{ Qualidade técnica do cuidado } \\
\hline Integridade física & A15 & Alívio da dor antes do procedimento & Equipe \\
\hline \multicolumn{4}{|l|}{ Durante o procedimento (D) } \\
\hline \multicolumn{4}{|l|}{ Acolhimento e orientação } \\
\hline \multirow[t]{2}{*}{ Agilidade } & D16 & Tempo de espera adequado & OMS \\
\hline & & (desde a chegada ao hospital até o procedimento) & \\
\hline \multicolumn{4}{|l|}{ Qualidade técnica do cuidado } \\
\hline Integridade física & D17 & Consciência preservada durante o procedimento (acordada ou dormindo) & OMS \\
\hline \multicolumn{4}{|l|}{ Qualidade técnica do cuidado } \\
\hline Qualificação profissional & D18 & Tipo de profissional que realizou o procedimento & OMS \\
\hline Acolhimento e orientação & & & \\
\hline Respeito * & D19 & Tratamento respeitoso (durante o procedimento) & OMS \\
\hline Acolhimento e orientação & & & \\
\hline Direito à informação & D20 & Receberam informação sobre o procedimento & OMS \\
\hline
\end{tabular}

(continua) 
Acolhimento e orientação

Direito à informação

Acolhimento e orientação

Direito à informação

Acolhimento e orientação Respeito *

Qualidade técnica do cuidado Integridade física

Qualidade técnica do cuidado Integridade física

Pós-procedimento $(P)$ **

Qualidade técnica do cuidado Integridade física

Qualidade técnica do cuidado Integridade física

Qualidade técnica do cuidado Qualificação profissional Respeito *

Acolhimento e orientação Privacidade

Acolhimento e orientação Privacidade

Acolhimento e orientação Privacidade

Continuidade do cuidado Cuidados pós-alta

Continuidade do cuidado Orientações sobre gravidez Continuidade do cuidado Consulta de revisão

Continuidade do cuidado Planejamento reprodutivo

Acolhimento e orientação

$$
\text { Respeito * }
$$

Acolhimento e orientação

$$
\text { Direito à informação }
$$

Qualidade técnica do cuidado Qualificação profissional

Continuidade do cuidado Acesso à contracepção

Continuidade do cuidado Acesso à contracepção

Continuidade do cuidado Acesso à contracepção

Continuidade do cuidado Acesso à contracepção
Entenderam a informação recebida sobre o procedimento

OMS

Tiveram interesse em saber sobre o procedimento

OMS

Confortadas pelo profissional durante o procedimento

OMS

Dor durante o procedimento

OMS

Alívio da dor durante o procedimento

OMS

Dor depois do procedimento

Equipe

Alívio da dor depois do procedimento

Equipe

P28 Tipo de profissional que realizou o exame depois do procedimento

OMS

OMS

Presença de pessoas durante o exame (depois do procedimento)

Equipe

Tipo de pessoas presentes durante o exame (depois do procedimento)

Equipe

Equipe exame depois do procedimento sobre planejamento reprodutivo 
Tabela 3 (continuação)

\begin{tabular}{|c|c|c|c|}
\hline $\begin{array}{l}\text { Momento assistencial/ } \\
\text { Dimensão/Critério }\end{array}$ & $\begin{array}{l}\text { Número } \\
\text { do item }\end{array}$ & Indicador & Fonte \\
\hline \multicolumn{4}{|l|}{ Impressão geral do atendimento } \\
\hline \multicolumn{4}{|l|}{ Insumos/Ambiente físico } \\
\hline Limpeza de roupa de cama & P44 & Troca suficiente de roupa de cama & Equipe \\
\hline \multicolumn{4}{|l|}{ Insumos/Ambiente físico } \\
\hline Limpeza do ambiente & P45 & $\begin{array}{l}\text { Limpeza das enfermarias e outros ambientes do hospital, } \\
\text { incluindo os banheiros }\end{array}$ & Equipe \\
\hline \multicolumn{4}{|l|}{ Insumos/Ambiente físico } \\
\hline Roupa adequada & P46 & Tamanho adequado de roupa fornecida & Equipe \\
\hline \multicolumn{4}{|l|}{ Insumos/Ambiente físico } \\
\hline Absorventes suficientes & P47 & Fornecimento suficiente de absorventes & Equipe \\
\hline \multicolumn{4}{|l|}{ Acolhimento e orientação } \\
\hline Apoio social & P48 & Presença de acompanhante durante a internação & Adaptado \\
\hline \multicolumn{4}{|l|}{ Acolhimento e orientação } \\
\hline Apoio social & P49 & Acompanhante permitido durante a internação & Adaptado \\
\hline \multicolumn{4}{|l|}{ Qualidade técnica do cuidado } \\
\hline Controle de pressão arterial & P50 & Medida de pressão arterial (antes e depois do procedimento) & Adaptado \\
\hline \multicolumn{4}{|l|}{ Qualidade técnica do cuidado } \\
\hline Controle de temperatura & P51 & Medida de temperatura (antes e depois do procedimento) & Adaptado \\
\hline \multicolumn{4}{|l|}{ Qualidade técnica do cuidado } \\
\hline Controle de sangramento & P52 & Avaliação de quantidade do sangramento (antes e depois do procedimento) & Equipe \\
\hline Avaliação global das distintas dimensões & P53 & Satisfação com o atendimento & Equipe \\
\hline \multicolumn{4}{|l|}{ Acolhimento e orientação } \\
\hline Respeito às diferenças (não discriminação) & P54 & Tratamento percebido como pior do que o dado a outras pacientes & Adaptado \\
\hline \multicolumn{4}{|l|}{ Acolhimento e orientação } \\
\hline Respeito às diferenças (não discriminação) & P55 & Tipo de discriminação percebida & Adaptado \\
\hline
\end{tabular}

OMS: Organização Mundial da Saúde.

Fontes: Organização Mundial da Saúde 8,12; Silva et al. 11.

* Respeito à condição de pessoa e/ou direito à escolha;

** Itens 33 ao 55 preenchidos obrigatoriamente após a mulher ter recebido alta médica. 


\section{Resumen}

En Brasil, hay poca investigación sobre la calidad de la atención al aborto inseguro. En este trabajo se presenta el primer paso en el desarrollo de un instrumento para evaluar la asistencia hospitalaria prestada por el Sistema Único de Salud en tres ciudades de Brasil. Junto con los criterios relacionados, se definieron cuatro dimensiones clave de la atención: acogida y orientación, calidad técnica del cuidado, continuidad de la atención, insumos/entorno físico. Realizamos una adaptación transcultural del conjunto de ítems propuestos por la Organización Mundial de la Salud. Tras una evaluación de las dimensiones y criterios no aprehendidos por este conjunto, se decidió adaptar preguntas de otros estudios y añadir otras desarrolladas por el equipo de investigación. El cuestionario fue pre-probado en tres ciudades con 52 usuarias para evaluar la aceptación y comprensión, tiempo de aplicación y ajustes finales. El instrumento totalizó 55 ítems, organizados de acuerdo a las etapas de la atención. Su uso más amplio depende de evaluaciones psicométricas posteriores, que están en marcha.

Aborto Inducido; Evaluación de Servicios de Salud; Cuestionários; Salud de la Mujer

\section{Referências}

1. Departamento de Ciência e Tecnologia, Secretaria de Ciência, Tecnologia e Insumos Estratégicos, Ministério da Saúde. Aborto e saúde pública no Brasil: 20 anos. Brasília: Ministério da Saúde; 2009. (Série B. Textos Básicos de Saúde).

2. Rocha BNGA, Uchoa SAC. Avaliação da atenção humanizada ao abortamento: um estudo de avaliabilidade. Physis (Rio J.) 2013; 23:109-27.

3. Aquino EML, Menezes G, Barreto-de-Araújo TV, Alves MT, Alves SV, Almeida MCC, et al. Qualidade da atenção ao aborto no Sistema Único de Saúde do Nordeste brasileiro: o que dizem as mulheres? Cienc Saúde Coletiva 2012; 17:1765-76.

4. Secretaria de Atenção à Saúde, Ministério da Saúde. Política nacional de atenção integral à saúde da mulher: plano de ação 2004-2007. Brasília: Ministério da Saúde; 2004. (Série C. Projetos, Programas e Relatórios).

5. Secretaria de Atenção à Saúde, Departamento de Ações Programáticas Estratégicas, Ministério da Saúde. Direitos sexuais e direitos reprodutivos: uma prioridade do governo. Brasília: Ministério da Saúde; 2005. (Série A. Normas e Manuais Técnicos) (Série Direitos Sexuais e Direitos Reprodutivos, Caderno 1).

\section{Colaboradores}

E. M. L. Aquino, G. M. S. Menezes, T. V. Barreto-de-Araújo, M. T. Alves, S. V. Alves, F. E. Schiavo, L. F. B. Marinho e L. C. Coimbra contribuíram na elaboração do protótipo, redação e aprovação do artigo. M. C. C. Almeida e M. E. Reichenheim colaboraram na redação e aprovação do artigo.

\section{Agradecimentos}

In memoriam de Luci Praciano Lima, parceira querida na realização deste estudo.
6. Secretaria de Atenção à Saúde, Ministério da Saúde. Atenção humanizada ao abortamento: norma técnica. Brasília: Ministério da Saúde; 2005. (Série A. Normas e Manuais Técnicos) (Série Direitos Sexuais e Direitos Reprodutivos, Caderno 4).

7. Lopes RM, Vieira-da-Silva LM, Hartz ZMA. Teste de uma metodologia para avaliar a organização, acesso e qualidade técnica do cuidado na atenção à diarréia na infância. Cad Saúde Pública 2004; 20 Suppl 2:S283-97.

8. Ministério da Saúde. Lei no 8.080, de 19 de setembro de 1990. Dispõe sobre as condições para a promoção, proteção e recuperação da saúde, a organização e o funcionamento dos serviços correspondentes e dá outras providências. Diário Oficial da União 1990; 20 set.

9. World Health Organization. Maternal and Health and Safe Motherhood Program. Studying unsafe abortion: a practical guide. Geneva: World Health Organization; 1996.

10. Reichenheim ME, Moraes CL. Operacionalização de adaptação transcultural de instrumentos de aferição usados em epidemiologia. Rev Saúde Pública 2007; 41:665-73. 
11. Silva AAM, Coimbra LC, Silva RA, Alves MTSSB, Lamy Filho F, Lamy ZC, et al. Perinatal health and mother-child health care in the municipality of São Luís, Maranhão State, Brazil. Cad Saúde Pública $2001 ; 17: 1413-23$.

12. World Health Organization. Pesquisa Mundial de Saúde.http://www.who.int/healthinfo/survey/whs longindividualapdf (acessado em 9/Jun/2012).

13. Ministério da Saúde. Pesquisa Nacional de Demografia e Saúde da Criança e da Mulher - PNDS 2006: dimensões do processo reprodutivo e da saúde da criança. Brasília: Ministério da Saúde, Centro Brasileiro de Análise e Planejamento, 2009. (Série G. Estatística e Informação em Saúde).

14. Vaitsman J, Andrade GRB. Satisfação e responsividade: formas de medir a qualidade e a humanização da assistência à saúde. Ciênc Saúde Coletiva 2005; 10:599-613.
15. Ciena AP, Gatto R, Pacini VC, Picanço VV, Magno IMN, Loth EA. Influência da intensidade da dor sobre as respostas nas escalas unidimensionais de mensuração da dor em uma população de idosos e de adultos jovens. Semina Ciênc Biol Saúde 2008; 29:12.

16. Mokkink LB, Terwee CB, Patrick DL, Alonso J, Stratford PW, Knol DL, et al. The COSMIN checklist for assessing the methodological quality of studies on measurement properties of health status measurement instruments: an international Delphi study. Qual Life Res 2010; 19:539-49.

Recebido em 21/Jan/2014

Versão final reapresentada em 15/Mai/2014

Aprovado em 22/Mai/2014 